\title{
Fractal dimensions of confined clusters in two-dimensional directed percolation
}

\author{
C Kaiser and L Turban \\ Laboratoire de Physique du Solidet, Université Henri Poincaré (Nancy I), BP 239, \\ F-54506 Vandouvre lès Nancy Cedex, France
}

Received 15 June 1994

\begin{abstract}
The fractal structure of directed percolation clusters, grown at the percolation threshold inside parabolic-like systems, is studied in two dimensions via Monte-Carlo simulations. With a free surface at $y= \pm C x^{k}$ and a dynamical exponent $z$, the surface shape is a relevant perturbation when $k<1 / z$ and the fractal dimensions of the anisotropic clusters vary continuously with $k$. Analytic expressions for these variations are obtained using a blob picture approach.
\end{abstract}

Since the work of Cardy [1], one knows that a large-scale alteration of the shape of a system may influence its local critical behaviour (further references can be found in the review [2]).

Cardy studied systems limited by corners or wedges which are scale invariant shapes for an isotropic system and thus, introduce a marginal perturbation leading to varying local exponents. More generally, one may consider systems limited by a parabolic-like surface at $y= \pm C x^{k}$ in the $(x, y)$-plane in two dimensions, a parabolic cylinder or a paraboloid in higher dimensions. Under a uniform rescaling of the lengths by a factor $b, C$ is changed into $C^{\prime}=b^{k-1} C$. As a consequence, $C$ grows to infinity for $k>1$ whereas it goes to zero when $k<1$. It remains scale invariant when $k=1$, which corresponds to the marginal corner geometry mentioned above.

Since $1 / C$ vanishes for the the semi-infinite system, it can be considered as a scaling field for the reference fixed point corresponding to the flat surface. This scaling variable is an irrelevant perturbation for $k>1$ and a relevant one for $k<1$.

In the relevant case, the shape renormalizes to a narrow system, so that the local order is weakened. Using a conformal transformation from the half-space to the interior of the parabola, one can show that the critical correlation functions decay from the tip along the $x$-axis in a stretched exponential way [3]. The order parameter at the tip also displays an essential singularity as a function of the reduced temperature $[3,4$, 5]. Similar results were obtained for the percolation problem [6]. In the case of chain polymers (self-avoiding-walks) with one end fixed at the tip, the exponent associated with the radius of gyration becomes anisotropic [7].

† Unité de Recherche Associée au CNRS No 155

cond-mat/9408076 

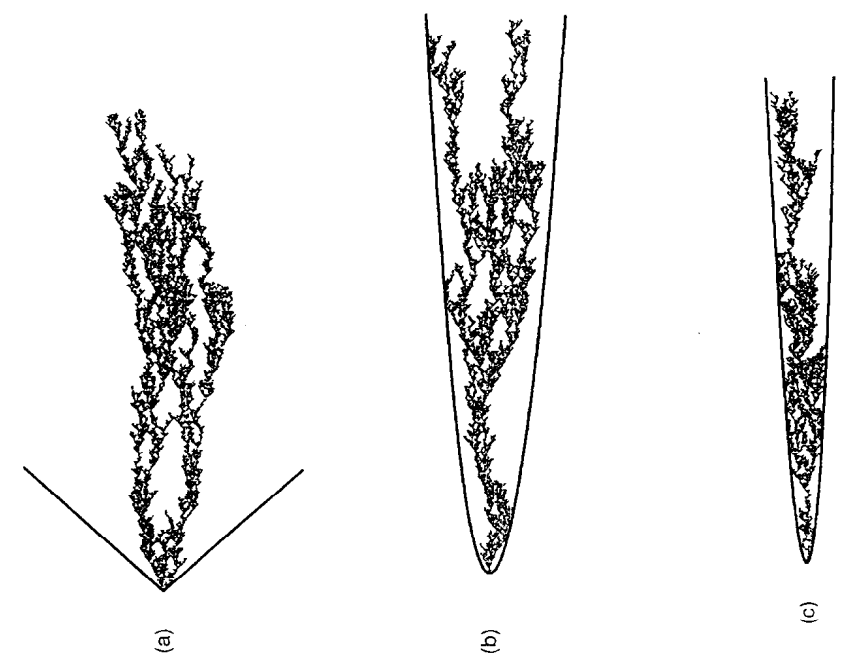

Figure 1. Structure of finite clusters generated at $p_{c}$ for: (a) free growth, $(\mathrm{b}-\mathrm{c}$ ) parabolic systems with $C=2,4$ and $k=1 / 2$.

When the unperturbed system is itself anisotropic, i.e. when the correlation length exponents $\nu_{\|}$in the $x$-direction and $\nu_{\perp}$ in the transverse direction are different with a dynamical exponent $z$ given by the ratio $\nu_{\|} / \nu_{\perp}$, the appropriate scaling factor becomes $b^{z}$ in the $x$-direction. Then the scaling field $1 / C$ transforms as

$$
\frac{1}{C^{\prime}}=b^{1-z k} \frac{1}{C}
$$

and the critical behaviour is modified when $k \leq 1 / z$ instead of 1 . The directed walk (polymer) with $z=2$ was studied in $[8,9]$. In the marginal case, which then corresponds to the true parabola, the local susceptibilities given by the numbers of walks with $N$ steps starting at the tip and ending either anywhere or on the surface, diverge with a $C$-dependent power of $N$ as expected.

In the present work, we study the structure of directed percolation clusters confined inside a "parabola". We consider the site percolation problem, directed along the diagonal of a square lattice, with the sites inside or on the "parabola" belonging to the system.

Using a standard Monte-Carlo method, $2.10^{5}$ clusters starting from the tip at $x=y=0$ were generated at the percolation threshold $p_{c}=0.705489(4)$ [10] as shown in figure 1. The square radii of gyration for $s$-site clusters along the two directions

$$
X_{s}^{2}=\frac{1}{2 s^{2}} \sum_{i, j=1}^{s}\left(x_{i}-x_{j}\right)^{2} \quad Y_{s}^{2}=\frac{1}{2 s^{2}} \sum_{i, j=1}^{s}\left(y_{i}-y_{j}\right)^{2}
$$

were put into bins with respect to the sizes $2^{i} \leq s<2^{i+1}(i=0,1,2, \ldots)$ and the bin averages $\left\langle X_{s}^{2}\right\rangle,\left\langle Y_{s}^{2}\right\rangle$, were calculated for "finite" clusters, i. e. for clusters with $x_{\max }<L=1000$, the system size along the $x$-direction. 


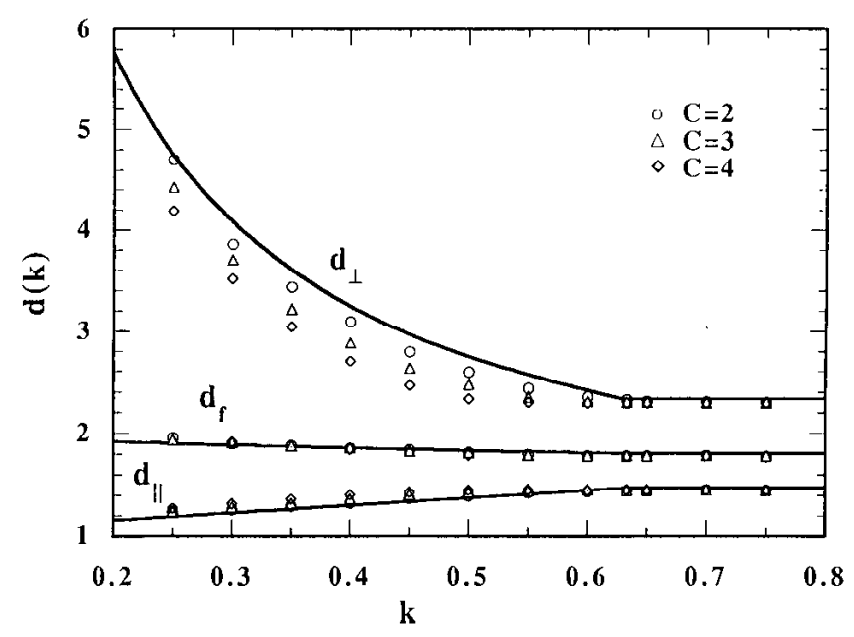

Figure 2. Fractal dimensions $d_{\|}(k), d_{\perp}(k)$ and $d_{f}(k)$ versus the system-shape exponent $k$. The lines give the analytic results obtained via a blob picture approach. The fractal dimensions are varying when the perturbation is relevant $(k<1 / z=$ $0.633(2))$.

Asymptotically, the following power laws are expected

$$
\bar{s} \sim{\overline{X_{s}}}^{d_{\|}(k)} \sim{\overline{Y_{s}}}^{d_{\perp}(k)}
$$

where $\bar{s}$ is the center of the bin, $\overline{X_{s}}=\left\langle X_{s}^{2}\right\rangle^{1 / 2}, \overline{Y_{s}}=\left\langle Y_{s}^{2}\right\rangle^{1 / 2}$ and $d_{\|}(k), d_{\perp}(k)$, are the fractal dimensions in the two directions. In a log-log plot, a linear behaviour is obtained for $2^{6}<\bar{s}<2^{12}-2^{13}$, the deviations at larger sizes being mainly due to the finite size of the system. There, the contribution from longer clusters with the same values of $\bar{s}$ is not taken into account. The fractal dimensions, shown in figure 2 versus $k$ for different values of $C$, were obtained from least-square fits of the data in the intermediate linear regime.

In order to analyse our results, let us first consider unconfined directed percolation [11]. In two dimensions, the best estimates for the critical exponents, obtained through series expansions, are [10]:

$$
\nu_{\perp}=1.097(2) \quad \nu_{\|}=1.734(2) \quad \gamma=2.278(2)
$$

giving

$$
z=1.581(3) \quad \beta=0.276(3)
$$

from scaling. Extending an argument of Stauffer for ordinary percolation [12], one obtains the following relations for the fractal dimensions of critical directed percolation clusters:

$$
d_{\|}=\frac{\beta+\gamma}{\nu_{\|}}=1.473(2) \quad d_{\perp}=\frac{\beta+\gamma}{\nu_{\perp}}=2.329(3)
$$




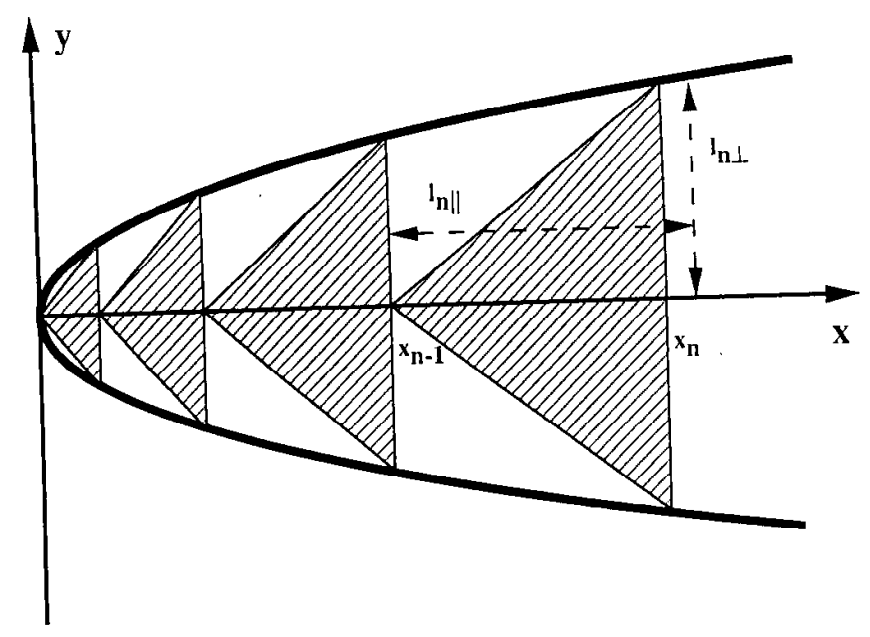

Figure 3. In the blob picture approach, confined clusters are built through piling up successive anisotropic blobs, with a transverse size $l_{n \perp}$ fixed by the geometry of the system. The correlations inside each blob are the same as for unconfined clusters.

The numerical value of $d_{\perp}>d$ may be at first sight surprising for a fractal object. Actually, it can be traced to the anisotropy. Let $l_{\|}$and $l_{\perp}$ be the length and width of a $s$-site cluster with $s \sim l_{\|}{ }_{\|} \sim_{l_{\perp}}^{d_{\perp}}$. It follows that

$$
l_{\|}=l_{\perp}{ }_{\perp} / d_{\|}=l_{\perp}^{z}
$$

grows faster with $s$ than $l_{\perp}$. $s$ itself grows faster with $l_{\perp}$ than an isotropic massive object due to the parallel growth. Introducing the characteristic length associated with the surface of the cluster

$$
l \sim\left(l_{\|} l_{\perp}\right)^{1 / 2} \sim s^{1 / d_{f}}
$$

a single fractal dimension $d_{f}$ can be defined with [11]

$$
\frac{1}{d_{f}}=\frac{1}{2}\left(\frac{1}{d_{\|}}+\frac{1}{d_{\perp}}\right)
$$

and now, $d_{f}=1.805(2)<2$.

When the perturbation introduced by the surface is relevant, the structure of confined clusters can be studied studied using a blob picture approach [13, 14, 7]. Inside the "parabola", the cluster configuration results from the piling up of anisotropic blobs as shown in figure 3. Within each blob, the correlations are the same as for unconfined clusters. The $n$th blob at $x_{n}$ has a width $l_{n \perp}=C x_{n}^{k}$ which is fixed by the geometry of the system. Using equation (7), its length is

$$
l_{n \|}=C^{z} x_{n}^{z k} \sim C^{u} n^{v}
$$


where the last expression corresponds to a continuum approximation in the blob index $n$. From $x_{n}-x_{n-1}=l_{n \|}$, one deduces the following differential equation in the continuum limit,

$$
\frac{\mathrm{d} x_{n}}{\mathrm{~d} n} \sim C^{u} n^{v} \sim C^{(u-z) / z k} n^{-1+v / z k}
$$

where the last term follows from the $n$-dependance of $x_{n}$ in (10). Identifying powers of $C$ and $n$, one obtains $u=z /(1-z k)$ and $v=k u$. These exponents are positive for a relevant perturbation only. Otherwise, when $k>1 / z$, the cluster grows freely, i. e. there is a single blob. Let $s_{n} \sim\left(l_{n \|}\right)^{d_{\|}}$be the number of sites inside the $n$th blob. For the whole cluster, we have:

$s=\sum_{n=1}^{N} s_{n} \sim C^{z d_{\|} /(1-z k)} \int_{1}^{N} \mathrm{~d} n n^{z k d_{\|} /(1-z k)} \sim C^{z d_{\|} /(1-z k)} N^{\left[1+z k\left(d_{\|}-1\right)\right] /(1-z k)}$.

In the same way, the cluster length is given by

$$
l_{\|}=\sum_{n=1}^{N} l_{n \|} \sim C^{z /(1-z k)} \int_{1}^{N} \mathrm{~d} n n^{z k /(1-z k)} \sim C^{z /(1-z k)} N^{1 /(1-z k)} .
$$

Eliminating $N$ between (12) and (13), one finally obtains:

$s \sim C^{z\left(d_{\|}-1\right)} l_{\|}^{d_{\|}(k)} \quad d_{\|}(k)=1+z k\left(d_{\|}-1\right) \quad 0 \leq k \leq 1 / z$

which, together with $l_{\perp}=C l_{\|}{ }^{k}$, also gives:

$s \sim C^{-1 / k} l_{\perp}^{d_{\perp}(k)} \quad d_{\perp}(k)=\frac{d_{\|}(k)}{k} \quad 0<k \leq 1 / z$.

The variations of the fractal dimensions are shown in figure 2. For a relevant perturbation, $d_{\|}(k)$ and $d_{\perp}(k)$ vary continuously with $k$ until they reach their unperturbed values for $k=1 / z$. As a result of finite-size effects, the Monte-Carlo values deviate from the analytical ones when $C$ increases. For large $C$ values the system is more open and the number of blobs inside it remains small for a finite system. As a consequence, the Monte-Carlo estimates for the fractal dimensions are then closer to the unperturbed ones.

Results for the tip percolation probability will be discussed elsewhere.

CK thanks Henri Poincaré University for hospitality. This work was supported by CNIMAT under project No 155/93.

\section{References}

[1] Cardy J L 1983 J. Phys. A: Math. Gen. 163617

[2] Iglói F, Peschel I and Turban L 1993 Adv. Phys. 42683

[3] Peschel I, Turban L and Iglói F 1991 J. Phys. A: Math. Gen. 24 L1229

[4] Davies B and Peschel I 1992 Ann. Phys. (Leipzig) 279

[5] Blawid S and Peschel I 1994 Z. Phys. B 9573 
[6] Berche B, Debierre J M and Eckle P 1994 Nancy I preprint

[7] Turban L and Berche B 1993 J. Physique I 3925

[8] Turban L 1992 J. Phys. A: Math. Gen. 25 L127

[9] Iglói F 1992 Phys. Rev. B 457024

[10] Essam J W, Guttmann A J and De'Bell K 1988 J. Phys. A: Math. Gen. 213815

[11] Kinzel W 1983 Ann. Israel Phys. Soc. 5425

[12] Stauffer D 1985 Introduction to Percolation Theory (London: Taylor \& Francis) p 64

[13] Pincus P 1976 Macromolecules 9386

[14] de Gennes P G 1979 Scaling Concepts in Polymer Physics (Ithaca: Cornell University Press) p 50 